\title{
Fraccionamiento químico del carbono en suelos con diferentes usos en el departamento de Magdalena, Colombia
}

\section{Chemical carbon fractioning in different land uses in the Magdalena Department, Colombia}

\author{
José Rafael Vásquez Polo ${ }^{1 *}$ y Felipe Macías Vázquez²
}

\footnotetext{
${ }^{1}$ Universidad del Magdalena. Carrera 32 No 22 - 08, Apartado Postal 2-1-21630. 470004 Santa Marta D.T.C.H., Colombia.

* Autor responsable (jose.quevas@yahoo.es)

${ }^{2}$ Universidad Santiago de Compostela. Facultad de Biología. Rúa Lope Gómez de Marzoa, s/n. (Campus Vida). 15782 Santiago de Compostela, España.
}

\section{RESUMEN}

El cambio en el uso y manejo de los suelos puede afectar su capacidad para capturar carbono, cuya magnitud es desconocida en la región norte del departamento de Magdalena, Colombia. En este sentido es importante avanzar en estudios de caracterización cualitativa de la materia orgánica del suelo y el efecto que sobre ella han tenido los cambios de uso y el manejo de suelos dedicados al monocultivo. Por tal motivo se estudió la relación de los diferentes usos del suelo sobre los contenidos y formas de carbono $(\mathrm{C})$ en seis zonas edafoclimáticas al norte de dicho departamento, ubicado entre 5 y $956 \mathrm{~m}$ de altitud, con temperaturas medias entre 24 y $30{ }^{\circ} \mathrm{C} \mathrm{y}$ precipitaciones medias entre 663 y $2000 \mathrm{~mm}$. En cada zona se muestrearon dos sitios, cultivos tropicales $\mathrm{y}$ bosques (húmedos $\mathrm{y}$ secos tropicales), tomando cuatro muestras compuestas por diez submuestras aleatorias por sitio, en las que se determinó $\mathrm{C}$ total $(\mathrm{Ct})$, C oxidable total (Cox), C hidrolizable con $\mathrm{HCl} 6 \mathrm{~N}$ (Ch), $\mathrm{C}$ ligado al material húmico $(\mathrm{Cp})$ mediante extracción con pirofosfato sódico y $\mathrm{C}$ no oxidable (Cnox) o $\mathrm{C}$ recalcitrante calculado como la diferencia Ct-Cox. El C total almacenado (Cta) por unidad de superficie se calculó teniendo en cuenta la densidad aparente (Da) y la profundidad de muestreo. Se encontraron diferencias estadísticas significativas por el efecto de las zonas, los valores mayores en porcentaje de $\mathrm{Nt}$ (0.32), Ct (3.90), Cox (3.85), Ch (2.05) y Cp (1.15) y Cta $\left(109 \mathrm{Mg} \mathrm{ha}^{-1}\right)$ se presentaron en los suelos de bosque húmedo de la zona 1 , siendo la de mayor altitud $(956 \mathrm{~m})$, precipitación $(2000 \mathrm{~mm})$ y menor temperatura $\left(24{ }^{\circ} \mathrm{C}\right)$. Para el factor usos del suelo solo presentaron diferencias significativas las propiedades: $\mathrm{Nt}, \mathrm{Ct}, \mathrm{Cox}$ y Ch. Los valores de las formas de C más estables para

Cita recomendada:

Vásquez Polo, J. R. y F. Macías Vázquez. 2017. Fraccionamiento químico del carbono en suelos con diferentes usos en el departamento de Magdalena, Colombia. Terra Latinoamericana 35: 7-17. los suelos de bosques, superaron los de los suelos de cultivo, en este contexto, Cox y Cnox representaron $95 \%$ y $5 \%$ del $\mathrm{Ct}$, respectivamente Se observó en promedio una pérdida de $26 \%$ del $\mathrm{Ct}$ en suelos de cultivo, en comparación con los suelos de bosques. El uso y manejo de los suelos cultivados en comparación a los suelos de bosque, influyó de manera negativa en los contenidos de las formas de $\mathrm{C}$ más estables.

Palabras clave: formas de carbono, fijación de carbono, almacenamiento de carbono.

\section{SUMMARY}

Land use change and management can affect the soil's ability to capture carbon; the magnitude of these changes is unknown in the northern region of the Magdalena Department, Colombia. For this reason, we studied the relationship of the different land uses to contents and forms of carbon in six soil and climate zones in northern Magdalena, Colombia, with altitudes between 5 and $956 \mathrm{~m}$, average temperatures between 24 and $30{ }^{\circ} \mathrm{C}$, and average rainfall between 663 and $2000 \mathrm{~mm}$. In each area, two sites were sampled: tropical crops and forests (Humid and Dry Tropical). Four samples consisting of ten random subsamples per site were taken. In each subsample, we determined total $\mathrm{C}(\mathrm{Ct})$, oxidizable $\mathrm{C}$ total (Cox), hydrolizable $\mathrm{C}$ with $6 \mathrm{~N} \mathrm{HCl}(\mathrm{Ch}), \mathrm{C}$ linked to humic material (Cp) by extraction with sodium pyrophosphate, rust free $\mathrm{C}$ (Cnox) or recalcitrant $\mathrm{C}$ calculated as the difference between $\mathrm{Ct}-\mathrm{Cox}$, total stored $\mathrm{C}$ (Cta) per unit of surface area, which is calculated by taking into account bulk density (Da) and sampling depth. Statistically significant differences were found for the effect of area; the highest percentages of $\mathrm{Nt}(0.32)$, 
Ct (3.90), Cox (3.85), Ch (2.05) and Cp (1.15) and Cta (109 $\left.\mathrm{Mg} \mathrm{ha}^{-1}\right)$ were found in soils of humid forest zone 1 with the highest altitude $(956 \mathrm{~m})$, precipitation $(2000 \mathrm{~mm})$ and the lowest temperature $\left(24^{\circ} \mathrm{C}\right)$. Land uses showed significant differences only for NT, CT, Cox, and $\mathrm{Ch}$. The values of the more stable forms of $\mathrm{C}$ of forest soils exceeded those of crop soils. Cox and Cnox accounted for $95 \%$ and $5 \%$ of $\mathrm{Ct}$, respectively, for the different land uses; an average loss of $26 \%$ of $\mathrm{Ct}$ was observed in cultivated soils as compared with forest soils.

Index words: carbon forms, carbon fixation, carbon stocks.

\section{INTRODUCCIÓN}

La materia orgánica del suelo (MOS) es termodinámicamente inestable bajo condiciones óxicas; su presencia en los suelos está explicada por la existencia de condiciones no ideales o de cinética oxidativa y metabólica ralentizada, de tal forma que el carbono (C) no es oxidado a $\mathrm{CO}_{2}$ (Albaladejo et al., 2013). La mayor reserva de $\mathrm{C}$ terrestre activo se encuentra en el suelo (Schlesinger y Bernhardt, 2013), con una cifra estimada de $2500 \mathrm{Pg}$ de $\mathrm{C}$ a 1 $\mathrm{m}$ de profundidad, comparado con $620 \mathrm{Pg}$ de la biota terrestre y detritos, $780 \mathrm{Pg}$ contenidos en la atmósfera y $39000 \mathrm{Pg}$ contenido en los océanos (Lal, 2010).

El aumento progresivo de la población mundial, proyectado en 9.2 billones para el año 2050, ha dado lugar a la conversión en gran escala de ecosistemas naturales a uso agrícola de la tierra; dicha conversión ha significado grandes emisiones de $\mathrm{C}$ a la atmósfera; los terrenos agrícolas contienen de 25 a $75 \%$ menos $\mathrm{C}$ orgánico (COS) que sus homólogos de los ecosistemas naturales (Lal, 2010). Las tasas actuales de pérdida de $\mathrm{C}$ debido al cambio en el uso del suelo son aproximadamente $1.6 \pm 0.8 \mathrm{Pg} \mathrm{C}$ año ${ }^{-1}$, cuyo efecto ocurre en los trópicos de manera más significativa (Smith, 2008). El uso tradicional del suelo y su gestión, han definido un gran sumidero de $\mathrm{C}$, estimado entre 50 a $100 \mathrm{Pg} \mathrm{C}$ en todos los suelos del mundo; no obstante, la restauración ecológica de la biosfera terrestre puede transferir algunas de las emisiones de $\mathrm{CO}_{2}$ de la atmósfera al stock de $\mathrm{C}$ terrestre y compensar también las emisiones antropogénicas (Lal, 2014). El stock de COS es extremadamente sensible a las perturbaciones tales como cambios en el uso del suelo, drenaje, labranza convencional y erosión. Por lo tanto, la adopción de prácticas sostenibles en el uso y manejo de los suelos son esenciales para minimizar la pérdida de C fijado en los suelos (Lal, 2014).

Gran parte de las emisiones de $\mathrm{C}$ a la atmósfera provienen de la deforestación. De acuerdo con los datos correspondientes al período 2002 al 2011 del IPCC (2013), se estima que anualmente se pierden por deforestación 13 millones de hectáreas de bosques tropicales, principalmente en Latinoamérica, lo cual produce globalmente una pérdida neta promedio de $0.9 \mathrm{Pg} \mathrm{C}$ año ${ }^{-1}(1 \mathrm{Pg}=1015 \mathrm{~g})$. El estudio del ciclo global del carbono (C) es prioritario para entender las consecuencias del incremento acelerado de la concentración de $\mathrm{CO}_{2}$ en la atmósfera, así como para diseñar estrategias a fin de reducir sus emisiones (Montaño et al., 2016), Los esfuerzos se han dirigido a estimar el contenido de $\mathrm{C}$ en los principales reservorios de los ecosistemas y los factores que determinan su almacenamiento y dinámica (Quéré et al., 2014).

En Colombia, los suelos dedicados a la agricultura convencional contienen de 1 a $3 \%$ de COS, mientras que los suelos de pastos y bosques por lo común contienen valores mayores (Mosquera, 2012). Guo y Gifford (2002) resaltaron la influencia que tenía el uso del suelo sobre los contenidos de COS; dichos autores, después de revisar 74 publicaciones, encontraron que los cambios de praderas a bosques artificiales, incluyendo plantaciones forestales, representaban pérdidas de $10 \%$ del $\mathrm{C}$ inicial, de bosques nativos a bosques artificiales pérdidas de $13 \%$, de bosques nativos a cultivos pérdidas de $42 \%$ y de praderas a cultivos pérdidas de $59 \%$ en el COS. En este contexto, la conversión de los usos del suelo a cultivos o monocultivos resultan casi siempre en pérdidas del COS (Stockmann et al., 2013).

El suelo puede actuar como un potencial sumidero de carbono, la acumulación de formas estables de $\mathrm{C}$ en el suelo depende de varios factores entre ellos el grado de complejidad de las moléculas húmicas (recalcitrancia bioquímica), su posición en los agregados del suelo (protección física) y el modo de asociación con metales y minerales edáficos secundarios (estabilización química); considerando estos mecanismos se ha comprobado que en muchos suelos hay formas de $\mathrm{C}$ no oxidables, incluso escapan a la oxidación con la técnica del dicromato en medio ácido y caliente. La aparición del "black carbón" y de estas formas recalcitrantes de $\mathrm{C}$ ponen en entre dicho que los datos del método de oxidación con dicromato sean los adecuados para 
calcular el sumidero total de $\mathrm{C}$ de los suelos y obligan a determinarlo por un método de mayores garantías como serían los de combustión a más de $1000{ }^{\circ} \mathrm{C}$ y en corriente rica en $\mathrm{O}_{2}$ determinando el $\mathrm{C}$ total a partir del $\mathrm{CO}_{2}$ desprendido. La diferencia entre el $\mathrm{C}$ total así obtenido y el $\mathrm{C}$ obtenido por oxidación ácida en caliente (Cox) podría utilizarse como una estimación del $\mathrm{C}$ no oxidable o más recalcitrante, llevando así una diferenciación de formas importantes desde el punto de vista del forzamiento climático (Macías et al., 2004).

Si la oxidación de la materia orgánica es una técnica que aprovecha su inestabilidad termodinámica, pueden realizarse otras determinaciones de formas de $\mathrm{C}$ basadas en propiedades diferentes. De esta manera, puede determinarse el $\mathrm{C}$ soluble en agua a diferentes temperaturas (Ghani et al., 2003); el $\mathrm{C}$ presente en la biomasa microbiana (Vance et al., 1987); el C metabolizable a diferentes condiciones de humedad (respirometrías), que puede obtenerse con los nutrientes actualmente existentes ( $\mathrm{C}$ metabolizable actual) o añadiendo nutrientes lábiles que incentiven el metabolismo de otras formas menos asimilables (C cometabolizable); el C hidrolizable en diferentes condiciones de $\mathrm{pH}$ (Parssons, 1989); el C mineralizado en tiempos de incubación determinados, con o sin adición de nutrientes; el $\mathrm{C}$ extraíble con diferentes agentes complejantes, como el $\mathrm{C}$ pirofosfato (Macías et al., 2004), que normalmente se considera equivalente al $\mathrm{C}$ humificado, $\mathrm{y}$ formas de $\mathrm{C}$ oxidables, que pueden obtenerse mediante agentes oxidantes con diferente capacidad oxidativa, p. ej: tetróxido de rutenio (Griffith et al., 1989). Tales agentes pueden incluir $\mathrm{KMnO}_{4}$ en diferentes concentraciones (Tirol- Padre y Ladha, 2004; diferentes condiciones de ataque con $\mathrm{K}_{2} \mathrm{Cr}_{2} \mathrm{O}_{7}$, oxidación con perboratos, persulfatos, hipoclorito, ozono, radicales $\mathrm{OH}^{-}$y otros, desde los más fácilmente oxidables hasta los no oxidables (Macías et al., 2004).

Mediante este trabajo se propuso evaluar la variación de los almacenes de $\mathrm{C}$ en sus distintas formas, asumiendo como hipótesis que el COS es afectado por los cambios en el uso del suelo, sin dejar de lado las pérdidas de $\mathrm{C}$ en suelos dedicados tradicionalmente a la agricultura intensiva.

\section{MATERIALES Y MÉTODOS}

La investigación se realizó al noreste del departamento de Magdalena (Figura 1), región considerada de alta tradición y vocación agropecuaria, con una importante área sembrada con cultivos de trascendencia económica para Colombia (IGAC, 2009).

Se realizó un muestreo aleatorio estratificado, con un diseño completamente al azar y un arreglo factorial con 2 factores, (1) zonas edafoclimáticas, con 6 niveles y (2) usos del suelo, con 2 niveles; en cada zona se seleccionaron dos sitios de muestreo, uno de cultivo y el otro en área de bosque, excepto en zona 4, donde se muestrearon 3 sitios (Cuadro 1). En cada sitio se tomaron cuatro muestras de suelos compuestas por 10 submuestras tomadas aleatoriamente en campo a profundidad 0 a $20 \mathrm{~cm}$ (Brady y Weil, 2002). La descripción de cada zona se llevó a cabo teniendo en cuenta el paisaje, clima ambiental, tipo de relieve y clasificación de suelos (Cuadro 1), descritos en el estudio de suelos y clasificación de tierras del departamento de Magdalena y Guajira, Colombia (IGAC, 2009).

Análisis del Carbono y Nitrógeno. El C total $(\mathrm{Ct})$ y $\mathrm{N}$ total $(\mathrm{Nt})$ se determinaron mediante un analizador LECO (Modelo CHN-1000, LECO Corp., St Joseph, $\mathrm{MI}$ ), para las formas oxidables de carbono presentes en el suelo se utilizó la metodología descrita por Macías et al. (2004); C oxidable total (Cox) mediante la técnica Walkley y Black (1934) en caliente $\left(105^{\circ} \mathrm{C}\right)$, $\mathrm{C}$ hidrolizable con $\mathrm{HCl} 6 \mathrm{~N}(\mathrm{Ch})$ descrito por Tan y Lal (2005); C humificado, ligado al material húmico (Cp)

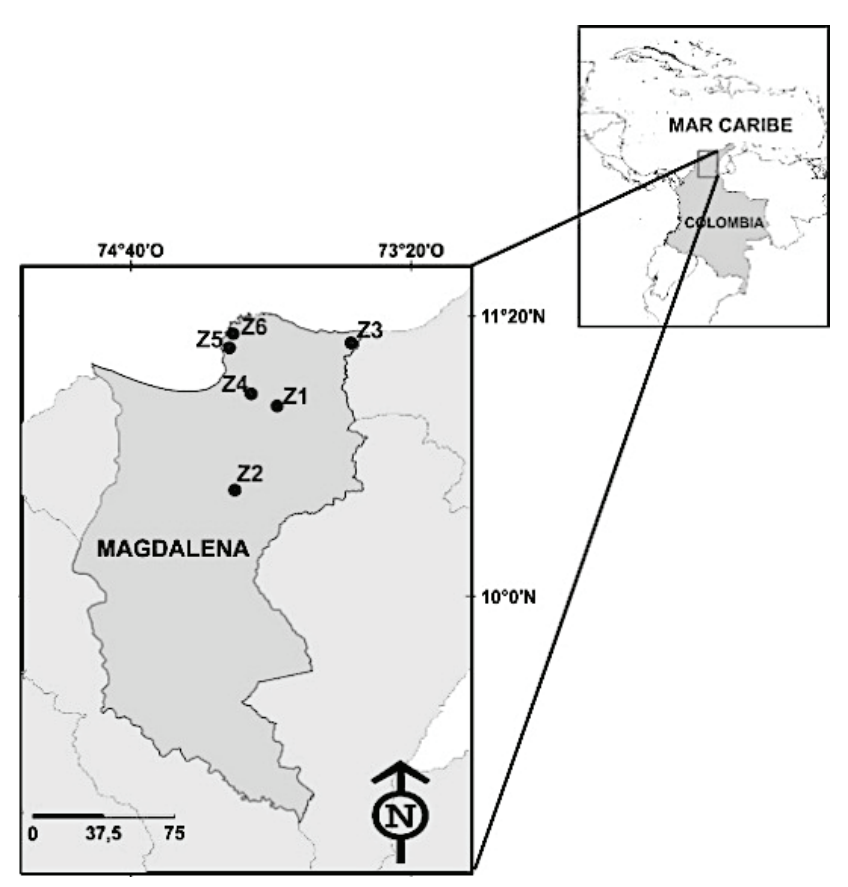

Figura 1. Localización del área de estudio. 
Cuadro 1. Descripción edafoclimática de las zonas de estudio.

\begin{tabular}{|c|c|c|c|c|c|}
\hline Zonas & $\begin{array}{l}\text { Sitios de } \\
\text { muestreo }\end{array}$ & Uso del suelo & Clima & Clasificación de suelos & Paisaje y material parental \\
\hline \multirow[t]{2}{*}{1} & 1 & $\begin{array}{l}\text { Cultivo de café con sombra, } \\
\text { anteriormente bosque húmedo } \\
\text { tropical }\end{array}$ & $\begin{array}{l}\text { Cálido húmedo; } \\
\text { Altitud } 956 \mathrm{~m} ; \\
\text { P } 2000 \mathrm{~mm} ; \\
\text { ETP } 990 \mathrm{~mm} ; \\
\text { T } 24{ }^{\circ} \mathrm{C}\end{array}$ & $\begin{array}{l}\text { Typic Eutrodepts } \\
\text { Údico Isohipertérmico } \\
\text { Cambisol Háplico }\end{array}$ & $\begin{array}{l}\text { Paisaje de montaña; } \\
\text { Ladera; } \\
\text { MP: esquistos }\end{array}$ \\
\hline & 2 & Bosque húmedo tropical & & & \\
\hline \multirow[t]{2}{*}{2} & 1 & $\begin{array}{l}\text { Cultivado hace más de } 20 \text { años } \\
\text { con palma africana, actualmente } \\
\text { se le dá un manejo orgánico } \\
\text { al cultivo y se alterna con } \\
\text { leguminosas como cobertura } \\
\text { para el suelo. }\end{array}$ & $\begin{array}{l}\text { Cálido seco; } \\
\text { Altitud 42m; } \\
\text { P } 1200 \mathrm{~mm} ; \\
\text { ETP } 1690 \mathrm{~mm} ; \\
\text { T } 28.4^{\circ} \mathrm{C}\end{array}$ & $\begin{array}{l}\text { Typic Ustifluvents } \\
\text { Ústico Isohipertérmico } \\
\text { Fluvisol Háplico }\end{array}$ & $\begin{array}{l}\text { Paisaje de planicie; } \\
\text { MP: sedimentos aluviales }\end{array}$ \\
\hline & 2 & Bosque seco tropical secundario & & & \\
\hline \multirow[t]{2}{*}{3} & 1 & $\begin{array}{l}\text { Suelo arado al momento del } \\
\text { muestreo para la siembra de } \\
\text { banano, anteriormente sembrado } \\
\text { con arroz. }\end{array}$ & $\begin{array}{l}\text { Cálido seco; } \\
\text { Altitud } 5 \mathrm{~m} \text {; } \\
\text { P } 1245 \mathrm{~mm} ; \\
\text { ETP } 1739 \mathrm{~mm} ; \\
\text { T } 27^{\circ} \mathrm{C}\end{array}$ & $\begin{array}{l}\text { Fluventic Haplustepts } \\
\text { Ústico Isohipertérmico } \\
\text { Cambisol Fluvéntico }\end{array}$ & $\begin{array}{l}\text { Paisaje de planicie; } \\
\text { MP: depósitos clásticos }\end{array}$ \\
\hline & 2 & Bosque seco tropical & & & \\
\hline \multirow[t]{3}{*}{4} & 1 & $\begin{array}{l}\text { Cultivo de banano tradicional, } \\
\text { anteriormente bosque seco } \\
\text { tropical }\end{array}$ & $\begin{array}{l}\text { Cálido seco; } \\
\text { Altitud } 15 \mathrm{~m} \text {; } \\
\text { P } 750 \mathrm{~mm} \text {; } \\
\text { ETP } 1890 \mathrm{~mm} \text {; } \\
\text { T } 27.5^{\circ} \mathrm{C}\end{array}$ & $\begin{array}{l}\text { Typic Haplustolls } \\
\text { Ústico Isohipertérmico } \\
\text { Phaozem Háplico }\end{array}$ & $\begin{array}{l}\text { Paisaje de planicie; } \\
\text { MP: sedimentos aluviales } \\
\text { medios }\end{array}$ \\
\hline & 2 & $\begin{array}{l}\text { Cultivo de mango hace más de } \\
15 \text { años. }\end{array}$ & & & \\
\hline & 3 & Bosque seco tropical secundario & & & \\
\hline \multirow[t]{2}{*}{5} & 1 & $\begin{array}{l}\text { Tradicionalmente sembrado } \\
\text { con frutales de clima cálido, } \\
\text { anteriormente bosque seco } \\
\text { tropical. }\end{array}$ & $\begin{array}{l}\text { Cálido muy seco; } \\
\text { Altitud } 10 \mathrm{~m} ; \\
\text { P } 880 \mathrm{~mm} ; \\
\text { ETP } 1910 \mathrm{~mm} ; \\
\text { T } 30^{\circ} \mathrm{C}\end{array}$ & $\begin{array}{l}\text { Fluventic Haplustepts } \\
\text { Ústico Isohipertérmico } \\
\text { Cambisol Fluvéntico }\end{array}$ & $\begin{array}{l}\text { Paisaje de planicie; } \\
\text { MP: sedimentos } \\
\text { fluviomarinos }\end{array}$ \\
\hline & 2 & Bosque muy seco tropical & & & \\
\hline \multirow[t]{2}{*}{6} & 1 & $\begin{array}{l}\text { Hortalizas de clima cálido, } \\
\text { anteriormente sembrados de } \\
\text { maíz y sorgo. }\end{array}$ & $\begin{array}{l}\text { Cálido muy seco; } \\
\text { Altitud } 7 \mathrm{~m} ; \\
\text { P } 663 \mathrm{~mm} ; \\
\text { ETP } 1986 \mathrm{~mm} ; \\
\text { T } 29^{\circ} \mathrm{C}\end{array}$ & $\begin{array}{l}\text { Typic Ustipsamments } \\
\text { Ústico Isohipertérmico } \\
\text { Arenosol Háplico }\end{array}$ & $\begin{array}{l}\text { Paisaje de planicie; } \\
\text { MP: sedimentos } \\
\text { fluviomarinos }\end{array}$ \\
\hline & 2 & Bosque muy seco tropical & & & \\
\hline
\end{tabular}

$\mathrm{T}=$ temperatura media anual; $\mathrm{P}=$ precipitación media anual; $\mathrm{ETP}=$ evapotranspiración potencial; $\mathrm{MP}=$ material parental.

mediante extracción con pirofosfato sódico (Velasco et al., 2010), para determinar los elementos metálicos por absorción atómica y el $\mathrm{C}$ digerido en solución ácida con dicromato potásico y cuantificado por valoración. A su vez, la fracción de C no oxidable (Cnox) fue calculada por la diferencia Ct-Cox; los contenidos de C total (Cta) por unidad de área se calcularon mediante la siguiente Ecuación 1:

$\mathrm{Cta}=\mathrm{Ct} * \mathrm{Da}^{*}$ Prof 
donde: Cta representa el almacenamiento de $\mathrm{C}$ en $\mathrm{kg} \mathrm{m}^{-2}$; Ct es $\mathrm{C}$ total en $\mathrm{g} \mathrm{kg}^{-1}$; Da es densidad aparente expresada en $\mathrm{g} \mathrm{cm}^{-3} \mathrm{y}$ prof es la profundidad de muestreo expresada en $\mathrm{m}$.

Análisis de la información. Para cada una de las propiedades evaluadas se estimaron los parámetros de tendencia central y de dispersión o variabilidad, con el fin de identificar aquellas variables con mayor poder de discriminación en relación al uso del suelo y zonas edafoclimáticas. Los valores de densidad aparente se determinaron por el método del cilindro, excepto en el sitio de cultivo de la zona 6 , donde se aplicó adicionalmente el procedimiento del terrón parafinado (IGAC, 2006). Se realizó un ANOVA factorial para establecer diferencias estadísticamente significativas entre zonas de muestreo, usos del suelo y su interacción. (Programa SPSS versión 22 de 2015).

\section{RESULTADOS Y DISCUSIÓN}

\section{Características de los Suelos Estudiados}

Se presentó un color en húmedo con matiz de 2.5YR en suelos de cultivo para las zonas 2,3 y 5 y en suelos de bosque zona 4; matiz de $10 \mathrm{YR}$ en todos los suelos de bosque excepto zona 4; correspondiendo a colores rojo-amarillentos oscuros, típicos de los horizontes superficiales influenciados por la presencia de pequeñas cantidades de materia orgánica. En relación a la textura, predominó la clase franco arcillo arenosa, con mayor porcentaje de la fracción arena, tanto para suelos de cultivo como para suelos de bosque. Los valores promedio obtenidos fueron de 1.35 y $1.43 \mathrm{~g} \mathrm{~cm}^{-3}$ para suelos de bosques y cultivo respectivamente (Cuadro 2), considerados normales a ligeramente altos para suelos de textura media (Malagón, 2003). Sin embargo, los valores mayores se presentaron en los suelos de cultivo, donde se expresó el efecto del laboreo de los suelos mediante el aumento de su densidad aparente, la cual se considera una propiedad sensible al cambio de uso.

Los valores promedio y las respectivas desviaciones estándares de las variables analíticas y formas de C determinadas en muestras de las zonas de muestreo se presentan en el Cuadro 2. La media del pH en los suelos cultivados fue 6.9 y de 7.1 para bosques, siendo la zona 1 (Cuadro 1) la que presentó los valores menores de $\mathrm{pH}$, de 5.6 y 6.5 respectivamente, marcados por la diferencia de altitud, pluviosidad y topografía del terreno, acorde a lo descrito en el Estudio General de Suelos y Zonificación de Tierras del Departamento del Magdalena (IGAC, 2009), para los suelos de montaña ubicados en las estribaciones de la Sierra Nevada de Santa Marta. En contraste con la zona 6, se observaron valores promedio de $\mathrm{pH}=8.6$ para suelos de cultivos $\mathrm{y}$ de 8.7 para suelos de bosques. Lo anterior se asocia con una alta saturación de bases en el complejo de cambio de los suelos estudiados, donde Ca es el catión dominante, el cual condiciona numerosos procesos fisicoquímicos (Vásquez et al., 2010). Por otro lado, se determinó una saturación media de 68 y $64 \%$ para los suelos de cultivo y bosques, respectivamente. Se detectaron diferencias estadísticas significativas entre zonas, a excepción de los contenidos de limo, destacando el efecto marcado de las condiciones edafoclimáticas (precipitación y temperatura, (Cuadro 1) propias de cada zona sobre las propiedades del suelo. El pH presentó diferencias significativas, detectándose evidencias del efecto de las labores de cultivo sobre la dinámica de dicha propiedad en los suelos estudiados (Jiménez et al., 2010). La relación $\mathrm{C} / \mathrm{N}$ presentó valores promedio de 10.7 para cultivos y de 11.4 en suelos de bosques, excepto zona 1 con valor medio de 12.5 en suelos de bosque y 9.8 en zona 3 suelo de cultivo; dichos valores bajo las condiciones del clima cálido tropical predominante hacen prevalecer los procesos de mineralización frente a los de humificación de la MOS (Vásquez et al., 2011).

\section{Análisis de las Formas y Contenidos de Carbono (C)}

El análisis de correlación de Pearson entre las propiedades físicas y químicas de los suelos con las formas de $\mathrm{C}$, observó una interacción significativa positiva entre los contenidos de Ca total y las formas Ct $(P=0.016)$ y $\operatorname{Cox}(P=0.024)$, lo que muestra la relevancia de dicho elemento en la estabilización de $\mathrm{C}$ en los suelos de estudio, lo que refleja su capacidad floculante (Almendros, 2004). De igual manera, los contenidos de $\mathrm{N}$ total $(\mathrm{Nt})$ presentaron alta significancia $\mathrm{y}$ se relacionaron positivamente con las diferentes formas de $\mathrm{C}$, lo cual evidencia una estrecha relación con compuestos nitrogenados, especialmente con el Ch $(\mathrm{r}=0.94)$, que en gran parte representa el $\mathrm{C}$ asociado a proteínas, acorde a lo descrito por Von Lützow et al. (2007). En contraste, se observó que el sodio (Na) total se correlaciona significativamente y con signo negativo con las formas de $\mathrm{C}$, lo que explica que altos contenidos de $\mathrm{Na}$ tienden a dispersar la materia orgánica y a 
Cuadro 2. Valores medios y las desviaciones estándares de las variables analíticas y formas de carbono determinadas en muestras de las diferentes zonas de estudio.

\begin{tabular}{|c|c|c|c|c|c|c|c|c|c|}
\hline Propiedades & Zona 1 & Zona 2 & Zona 3 & Zona 4 & Zona 5 & Zona 6 & Media & $\mathrm{CV}$ & DS \\
\hline \multicolumn{10}{|c|}{ Cultivo } \\
\hline $\mathrm{Da}$ & $1.49 \mathrm{ab}$ & $1.4 \mathrm{bcd}$ & $1.25 \mathrm{de}$ & $1.57 \mathrm{a}$ & $1.43 \mathrm{bc}$ & $1.45 \mathrm{abc}$ & 1.45 & 8.8 & 0.13 \\
\hline $\mathrm{pH}$ & $5.6 \mathrm{~d}$ & $6.3 \mathrm{~cd}$ & $6.5 \mathrm{~cd}$ & $7.1 \mathrm{bc}$ & $7.1 \mathrm{bc}$ & $8.6 \mathrm{a}$ & 6.86 & 13.47 & 0.93 \\
\hline $\mathrm{Nt}(\%)$ & $0.23 \mathrm{abc}$ & $0.12 \mathrm{c}$ & $0.13 \mathrm{bc}$ & $0.17 \mathrm{bc}$ & $0.13 \mathrm{c}$ & $0.12 \mathrm{c}$ & 0.15 & 32.95 & 0.05 \\
\hline $\mathrm{Ct}(\%)$ & $2.61 \mathrm{abc}$ & $1.343 \mathrm{c}$ & $1.29 \mathrm{c}$ & $1.84 \mathrm{bc}$ & $1.28 \mathrm{c}$ & $1.28 \mathrm{c}$ & 1.61 & 37.84 & 0.62 \\
\hline $\mathrm{Ch}(\%)$ & $1.43 \mathrm{abc}$ & $0.79 \mathrm{c}$ & $0.66 \mathrm{c}$ & $0.88 \mathrm{c}$ & $0.74 \mathrm{c}$ & $0.79 \mathrm{c}$ & 0.88 & 36.86 & 0.32 \\
\hline Cp (\%) & $0.81 \mathrm{abc}$ & $1.00 \mathrm{ab}$ & $0.59 \mathrm{bcd}$ & $0.53 \mathrm{~cd}$ & $0.18 \mathrm{~d}$ & $0.45 \mathrm{~cd}$ & 0.59 & 48.98 & 0.28 \\
\hline Cnox $(\%)$ & $0.05 \mathrm{a}$ & $0.04 \mathrm{a}$ & $0.14 \mathrm{a}$ & $0.10 \mathrm{a}$ & $0.06 \mathrm{a}$ & $0.02 \mathrm{a}$ & 0.06 & 157.5 & 0.12 \\
\hline $\operatorname{Cox}(\%)$ & $2.57 \mathrm{abc}$ & $1.307 \mathrm{c}$ & $1.15 \mathrm{c}$ & $1.74 \mathrm{bc}$ & $1.23 \mathrm{c}$ & $1.26 \mathrm{c}$ & 1.54 & 40.26 & 0.63 \\
\hline $\mathrm{C} / \mathrm{N}$ & $11.5 \mathrm{a}$ & $11.3 \mathrm{a}$ & $9.8 \mathrm{a}$ & $10.9 \mathrm{a}$ & $10.3 \mathrm{a}$ & $10.5 \mathrm{a}$ & 10.69 & 11.87 & 1.27 \\
\hline \multicolumn{10}{|c|}{ Bosque } \\
\hline $\mathrm{Da}$ & $1.4 \mathrm{bcd}$ & $1.33 \mathrm{cde}$ & $1.19 \mathrm{e}$ & $1.46 \mathrm{abc}$ & $1.35 \mathrm{bcd}$ & $1.36 \mathrm{bcd}$ & 1.35 & 7.21 & 0.1 \\
\hline $\mathrm{pH}$ & $6.5 \mathrm{~cd}$ & $5.6 \mathrm{~d}$ & $7.0 \mathrm{bc}$ & $7.8 \mathrm{ab}$ & $7.1 \mathrm{bc}$ & $8.7 \mathrm{a}$ & 7.13 & 15 & 1.07 \\
\hline $\mathrm{Nt}(\%)$ & $0.32 \mathrm{a}$ & $0.17 \mathrm{bc}$ & $0.14 \mathrm{bc}$ & $0.15 b c$ & $0.25 \mathrm{ab}$ & $0.15 b c$ & 0.19 & 44.11 & 0.08 \\
\hline $\mathrm{Ct}(\%)$ & $3.90 \mathrm{a}$ & $1.64 \mathrm{bc}$ & $1.72 \mathrm{bc}$ & $1.76 \mathrm{bc}$ & $3.26 \mathrm{ab}$ & $1.62 \mathrm{bc}$ & 2.32 & 55.6 & 1.29 \\
\hline $\mathrm{Ch}(\%)$ & $2.05 \mathrm{a}$ & $0.93 \mathrm{bc}$ & $0.88 \mathrm{bc}$ & $0.82 \mathrm{c}$ & $1.81 \mathrm{ab}$ & $0.87 \mathrm{c}$ & 1.23 & 55.42 & 0.68 \\
\hline Cp (\%) & $1.15 \mathrm{a}$ & $0.633 \mathrm{bcd}$ & $0.67 \mathrm{bc}$ & $0.34 \mathrm{~cd}$ & $0.78 \mathrm{abc}$ & $0.49 \mathrm{~cd}$ & 0.68 & 51.96 & 0.35 \\
\hline Cnox $(\%)$ & $0.05 \mathrm{a}$ & $0.02 \mathrm{a}$ & $0.09 \mathrm{a}$ & $0.13 \mathrm{a}$ & $0.04 \mathrm{a}$ & $0.10 \mathrm{a}$ & 0.07 & 109.06 & 0.07 \\
\hline Cox $(\%)$ & $3.85 \mathrm{a}$ & $1.62 \mathrm{bc}$ & $1.64 \mathrm{bc}$ & $1.63 \mathrm{bc}$ & $3.22 \mathrm{ab}$ & $1.52 \mathrm{bc}$ & 2.25 & 58.14 & 1.3 \\
\hline $\mathrm{C} / \mathrm{N}$ & $12.5 \mathrm{a}$ & $9.75 \mathrm{a}$ & $11.8 \mathrm{a}$ & $12.0 \mathrm{a}$ & $12.5 \mathrm{a}$ & $10.3 \mathrm{a}$ & 11.46 & 19.25 & 2.25 \\
\hline
\end{tabular}

$\overline{\mathrm{CV}}=$ coeficiente de variación; $\mathrm{DS}=$ desviación estándar; medias con distintas letras entre filas y columnas son diferentes entre sí: Tukey $(P \leq 0.05)$. $\mathrm{Da}=$ densidad aparente; $\mathrm{Nt}=$ nitrógeno total $\mathrm{Ct}=$ carbono total; $\mathrm{Ch}=$ carbono hidrolizable $\mathrm{Cp}=$ carbono pirofosfato; $\mathrm{Cnox}=$ carbono no oxidable .

dificultar la producción de biomasa y necromasa. Por otro lado, los suelos con $\mathrm{pH}$ mayores a 7.5 encontrados en las zonas 4 y 6 observaron los menores contenidos de carbono total. (Cuadro 2).

A excepción del Cnox y la relación $\mathrm{C} / \mathrm{N}$, las demás formas de $\mathrm{C}$ estudiadas presentaron diferencias estadísticamente significativas entre zonas; los mayores contenidos se encontraron en los suelos de bosques de la zona 1 (Cuadro 2), donde las condiciones edafoclimáticas favorecen las entradas de la $\mathrm{MO}$ al suelo (Vásquez et al., 2011). Para el factor usos del suelo, solo se detectaron diferencias significativas en las variables $\mathrm{Nt}, \mathrm{Ct}, \mathrm{Cox}$ y $\mathrm{Ch}$; la tendencia global sugiere que los valores de las formas de $\mathrm{C}$ para los suelos de bosques superaron los de suelos de cultivos, en contraste con los datos de la zona 4, donde los porcentajes de $\mathrm{Ct}$, Cox, Ch y $\mathrm{Cp}$ de los suelos de cultivos superaron a los obtenidos en los suelos de bosques. Lo anterior se debe posiblemente al manejo agronómico del cultivo de banano, que implica un aporte alto de material vegetal con una baja relación $\mathrm{C} / \mathrm{N}$, asimismo, al cultivo de mango, en el cual la hojarasca depositada en la superficie del suelo afecta notablemente la dinámica de descomposición de la MO. Los valores de Cnox para la zona 4 fueron mayores en los suelos de bosques, comprobando su efectividad para la captura de $\mathrm{C}$, aunque es previsible el consecuente deterioro de la misma por el cambio en el uso del suelo (Cuadro 2).

La concentración de $\mathrm{C}$ total estuvo por debajo del $3 \%$ en los suelos de cultivo y bosque de las zonas 2 , 3, 4 y 6 (Cuadro 2); sólo los suelos con vegetación de bosque húmedo de la zona 1 y del bosque seco en la zona 5 superaron el $3 \%$ de $\mathrm{C}$ total, valor que se considera es el mínimo para disponer de una adecuada estabilidad 
Cuadro 3. Almacenamiento de las diferentes formas de $\mathrm{C}\left(\mathrm{Mg} \mathrm{ha}^{-1}\right)$ a $20 \mathrm{~cm}$ de profundidad.

\begin{tabular}{llllllll}
\hline Formas de C & Zona 1 & Zona 2 & Zona 3 & Zona 4 & Zona 5 & Zona 6 & Media \\
\hline Ct & 78.1 & 37.6 & 32.1 & 58.0 & 36.6 & 36.9 & 46.6 \\
$\mathrm{Ch}$ & 42.8 & 22.2 & 16.5 & 27.6 & 21.0 & 22.8 & 25.5 \\
$\mathrm{Cp}$ & 24.1 & 28.1 & 14.7 & 16.7 & 5.1 & 13.0 & 16.9 \\
$\mathrm{Cnox}$ & 1.3 & 1.0 & 3.4 & 3.1 & 1.6 & 0.5 & 1.8 \\
$\mathrm{Cox}$ & 76.8 & 36.6 & 28.7 & 54.8 & 35.0 & 36.4 & 44.7 \\
& & & & Cultivo & & \\
$\mathrm{Ct}$ & 109.1 & 43.7 & 40.9 & 51.1 & 88.1 & 43.8 & 62.8 \\
$\mathrm{Ch}$ & 57.4 & 24.8 & 20.9 & 23.9 & 48.8 & 23.5 & 33.2 \\
$\mathrm{Cp}$ & 32.2 & 16.9 & 15.8 & 10.0 & 21.1 & 13.2 & 18.2 \\
$\mathrm{Cnox}$ & 1.5 & 0.5 & 2.0 & 3.6 & 1.1 & 2.6 & 1.9 \\
Cox & 107.6 & 43.2 & 39.0 & 47.5 & 87.0 & 41.2 & 60.9 \\
\hline
\end{tabular}

$\mathrm{Ct}=$ carbono total $; \mathrm{Ch}=$ carbono hidrolizable $; \mathrm{Cp}=$ carbono pirofosfato Cnox = carbono no oxidable; $\mathrm{Cox}=$ carbono oxidable total.

estructural, según la Estrategia Europea de Protección del Suelo (Macías et al., 2004). Al considerar dichos valores dentro del contexto de los suelos colombianos y según el IGAC (2009) quien estableció como rangos medios de $\mathrm{C}$ para el clima cálido valores entre 1.5 y $2 \%$ resultan no ser excesivamente bajos, aunque sí hacen evidentes los problemas de erosión, baja capacidad de intercambio y baja retención de humedad que pueden aparecer asociados a las pocas entradas de materiales orgánicos y a las altas tasas de mineralización de la materia orgánica en estos suelos, asimismo, la escasa relevancia de estos ecosistemas, incluyendo los naturales, como sumideros de $\mathrm{C}$.

Los contenidos de $\mathrm{Ct}$ en relación a los usos del suelo fueron significativamente diferentes $(P \leq 0.05)$ (Cuadro 2), con excepción de la zona 4, con cultivo de banano y mango, cuyo valor de $\mathrm{Ct}(1.84 \%)$ fue superior al de los suelos de bosques para la misma zona $(1.76 \%)$. Dicho incremento en el cultivo del banano y mango puede explicarse por la cantidad importante de aportes de material orgánico que se realizan en estos cultivos. Al respecto, los resultados deben ser tomados en cuenta en la gestión agronómica, hacen evidente que las adiciones de residuos verdes en los suelos cultivados permite mantener e incluso aumentar ligeramente el sumidero de $\mathrm{C}$ en las condiciones semiáridas. En sentido negativo, también ponen de manifiesto que determinadas actuaciones, como las que se han llevado a cabo en la transformación en cultivo de frutales (zona 5), pueden reducir drásticamente (50 a 60\%) el contenido de $\mathrm{C}$ en los primeros $20 \mathrm{~cm}$ de suelo. Un estudio más específico de la historia del cultivo en esta zona permitirá elaborar recomendaciones que eviten procesos de degradación.

Respecto a las formas de C, se observó escaso C recalcitrante; en los suelos de bosques, el porcentaje de esta forma de $\mathrm{C}$ de elevada persistencia, equivalente al black carbón, representa 1 a $7 \%$ con máximo en el bosque seco de la zona 4 , mientras que en los suelos cultivados representa de 1 a $11 \%$, con máximo en el suelo arado para siembra de banano en la zona 3. En la mayor parte de los suelos el $\mathrm{C}$ recalcitrante suele formarse a través de los incendios de alta intensidad, por medio de transformaciones pirolíticas que incrementan la concentración de moléculas aromáticas, aunque otros procesos pueden estar implicados. El bajo porcentaje de esta fracción tiene consecuencias importantes, toda vez que el $\mathrm{C}$ recalcitrante es relativamente inerte frente al metabolismo y la oxidación, aunque ejerce importantes actuaciones físico-químicas dentro del suelo por su capacidad de retención de agua, iones, nutrientes, contaminantes, su integración con la actividad biótica y la formación de agregados estables (Macías et al., 2004).

El C oxidable es la fracción que representa $90 \%$ o más del C total. Es cierto que el método de oxidación utilizado es el más drástico en capacidad 
oxidativa $\left(\mathrm{K}_{2} \mathrm{Cr}_{2} \mathrm{O}_{7}\right.$ en medio ácido y caliente, con un Eh > 1000 mvol) y que dichas condiciones no se pueden producir en los suelos por lo que este elevado porcentaje no refleja adecuadamente el comportamiento de la materia orgánica de los suelos frente a los procesos oxidativos y metabólicos que se producen en ellos. Aun así, resulta evidente que el método oxidativo proporciona un valor útil para la estimación del C total y que en condiciones drásticas prácticamente toda la materia orgánica podría ser oxidada o metabolizada.

$\mathrm{El} \mathrm{Ch}$ en $\mathrm{HCl} 6 \mathrm{~N}$ el cual remueve eficientemente el $\mathrm{C}$ asociado a carbohidratos, proteínas, hemicelulosa y a complejos órgano-minerales, representó el $60 \%$ del Cox en suelos de cultivo y $57 \%$ en suelos de bosques, 10 cual coincide con los valores publicados por Stevenson (1994) y Paul et al. (2006), quienes reportaron que el Ch con HCL $6 \mathrm{~N}$ representa de 30 a $87 \%$ del COS. Al tomar en cuenta las formas de $\mathrm{C}$ representadas por el Ch y los porcentajes citados anteriormente, para suelos de cultivo y bosques, puede afirmarse que los suelos de bosques poseen un mayor porcentaje de $\mathrm{C}$ asociado a formas de menor labilidad, posiblemente porque están presentes mayores cantidades de formas con mayor estabilidad biológica, lo cual incluye materiales con grupos alquilos, arilos, lignina y celulosa cristalina (Von Lützów et al., 2007). El Ch representa cifras alrededor de $50 \%$ del $\mathrm{Ct}$ en ambos tipos de usos (47 a $57 \%$ en suelos de bosque y 48 a $62 \%$ en los cultivados), de formas de $\mathrm{C}$ correspondientes al $\mathrm{Ch}$ de carbohidratos y proteínas, es decir, fundamentalmente ligado a las formas orgánicas más abundantes en la necromasa fresca, las cuales son rápidamente metabolizables, lo que sugiere un valor alrededor del $40 \%$ como estimación de las formas más difícilmente metabolizables y oxidables.

Finalmente, el porcentaje de $\mathrm{Cp}$, que suele identificarse con el C humificado existente, se ubicó de 19 a $31 \%$ del Ct en los suelos con vegetación natural y más variable en los cultivados (14-71\%), siendo mínimo en el cultivo de frutales y máximo en el de palma, lo que se relaciona con las diferencias de aporte y humificación existentes entre dichos sistemas. Se presentaron valores bajos de $\mathrm{Cp}$, lo que insinúa poca formación de complejos orgánicos, al parecer debido al predominio de humus joven con baja capacidad para la formación de complejos (Shoji y Fujiwara, 1984).

En general, los resultados obtenidos coinciden con estudios de Moreno y Lara (2003) en Colombia, quienes trabajaron en Inceptisoles y Entisoles del departamento de Antioquia. Dichos autores encontraron que el COS de los bosques primarios fue superior al de suelos de áreas intervenidas y de los bosques secundarios. Sin embargo, en estudios realizados por Carvajal et al. (2009), quienes estudiaron suelos con diferentes usos en paisajes andinos colombianos, mostraron una tendencia diferente en diferentes perfiles $(0-30 \mathrm{~cm})$. Observaron que los suelos presentaron ganancias en el almacenamiento de $\mathrm{C}$ cuando las coberturas vegetales naturales, a partir de relictos de selva, se transformaron en cultivos, principalmente de café y plátano, mientras que se notaron disminuciones considerables cuando los cultivos se convirtieron en pastizales.

\section{Acumulación de Carbono}

Se muestra, en el Cuadro 3, la tendencia de almacenamiento de las distintas formas de $\mathrm{C}$ a $20 \mathrm{~cm}$ de profundidad en las diferentes zonas, al tomar en cuenta los usos del suelo.

La acumulación media de las formas de C estudiadas presentó mayores valores en los suelos de bosques, comparados con suelos de cultivo para una misma zona; con valores medios de $\mathrm{Ct}$ en suelos de bosque alrededor de los $63 \mathrm{Mg} \mathrm{ha}^{-1}$, por debajo de los $99 \mathrm{Mg} \mathrm{ha}^{-1}$ planteados en el estudio de Lorenz y Lal (2014) de estimación media del COS para diferentes biomas en el mundo. No obstante, en la zona 4 los valores de Ct, $\mathrm{Ch}, \mathrm{Cox}$ y $\mathrm{Cp}$ fueron mayores en los suelos de cultivo, debido posiblemente al intenso retorno de material vegetal al suelo en el manejo agronómico del cultivo de banano. Ridley et al. (1990), en Australia encontraron $150 \%$ más de $\mathrm{C}$ en suelos de cultivo respecto a suelos nativos; tales situaciones aparentemente anómalas, en las que el sistema más intensificado agronómicamente contiene más $\mathrm{C}$, pueden producirse por causas específicas de manejo que incrementan el retorno de mayores cantidades de biomasa y necromasa al suelo, sea por un aporte externo o por un incremento de la producción asociado a la aplicación de fertilizantes. Lo anterior concuerda con las observaciones de Ibrahim et al. (2007), quienes encontraron que niveles de $C$ en suelos con praderas nativas fueron superados por los cultivados en la zona; en el mismo sentido, Mosquera et al. (2012) encontraron almacenes de $\mathrm{C}$ mayores en suelos de pradera que en suelos forestales nativos en la Amazonía colombiana. En el presente estudio, 
las formas de C más estables fueron siempre mayores en suelos de bosques, que tienen mayor capacidad natural para capturar y estabilizar más C (Macías et al., 2004).

Las condiciones climáticas de la zona 1, en particular las diferencias derivadas de la mayor altitud sobre el nivel del mar con relación a las demás zonas de muestreo (Cuadro 1), condicionaron la mayor acumulación de $\mathrm{C}$, acorde a lo descrito por Malagón et al. (2003) y en concordancia con lo expuesto por Carvajal et al. (2009). La mayor acumulación de la forma de $\mathrm{C}$ ligada a la fracción húmica $(\mathrm{Cp})$ se presentó en la zona 2, donde prospera el cultivo de Palma Africana (Cuadro 1) influenciado por el manejo orgánico asociado al cultivo, con un alta adición de formas lábiles de C; dicho a su vez este cultivo presentó la menor capacidad para capturar $\mathrm{C}$ recalcitrante expresado por la baja acumulación de la forma Cnox.

El análisis interior de cada zona permitió establecer las pérdidas de $\mathrm{Ct}$ en relación al cambio de uso del suelo, tomando como referencia el $\mathrm{C}$ almacenado en los suelos de bosques. Six et al. (2002) analizaron el concepto de "saturación de C" introducido por Hassink (1997), al considerar que los niveles naturales de $\mathrm{C}$ de los suelos reflejan el balance de ganancias $\mathrm{y}$ pérdidas de $\mathrm{C}$ teniendo en cuenta factores como la productividad y los regímenes de humedad y temperatura, no necesariamente representan el límite superior de la capacidad de almacenamiento de $\mathrm{C}$ del suelo. Lo anterior significa que "muchos suelos, si no todos, podrían almacenar más $\mathrm{C}$ en forma metaestable del que tienen en condiciones naturales. El Ct y Cox se perdieron en los suelos de cultivo, con relación a los suelos de bosque, en promedio para todas las zonas aproximadamente $16 \mathrm{Mg} \mathrm{ha}^{-1} \mathrm{C}$, lo cual representa una pérdida de $26 \%$; a su vez, una pérdida media de 7.7 $\mathrm{Mg} \mathrm{ha}^{-1}$ de $\mathrm{Ch}$, que corresponde a un $23.3 \%$. Lo anterior concuerda con lo descrito por Calvo de Anta (1992) en un estudio realizado en suelos de la provincia de Coruña (España), donde se comprobó que los suelos de cultivos tenían pérdidas de $\mathrm{C}$ en relación a los suelos de bosque, que oscilaban de 26 a 49\%, siendo máximas en los suelos sobre esquistos y anfibolitas y menores en los de gabros. Se estimaron asimismo pérdidas de 1.2 $\mathrm{Mg} \mathrm{ha}^{-1}$ de $\mathrm{Cp}$ equivalentes a $6.8 \%$; pérdidas de Cnox de $0.1 \mathrm{Mg} \mathrm{ha}^{-1}$ equivalentes a $4 \%$ y en relación a la fracción Cdox, pérdidas de $15 \mathrm{Mg} \mathrm{ha}^{-1}$ o $35 \%$ de C. Si se relacionan las pérdidas de $\mathrm{C}$ con los suelos cultivados, se observa que el suelo plantado con café presentó pérdidas de $28.4 \%$ de Ct, lo cual concuerda con lo descrito por Hoyos et al. (2005); a su vez, el cultivo de la Palma africana indujo pérdidas de $15.3 \%$, mientras que los cultivos de banano y mango experimentaron ganancias de aproximadamente 15\% de $\mathrm{Ct}$, Cox, y $\mathrm{Ch}$ y d $67 \%$ para $\mathrm{Cp}$, aunque para las formas recalcitrantes de Cnox presentaron pérdidas de $13.5 \%$.

\section{CONCLUSIONES}

- La forma oxidable del carbono (C) representó 95\% del $\mathrm{C}$ total para los diferentes usos del suelo, independiente de la zona edafoclimática. Los almacenes de C disminuyeron de manera directamente proporcional a la altura sobre el nivel del mar, observándose una marcada diferencia entre la zona 1 (956 m de altitud) y las demás zonas estudiadas, ubicadas por debajo de los $42 \mathrm{~m}$ de altitud.

- El uso y manejo de los suelos cultivados comparados con los suelos de bosques, influyó negativamente en los contenidos de las formas de C más estables, toda vez que en promedio se detectaron pérdidas de $\mathrm{C}$ total alrededor de $26 \%$. Es importante conseguir un incremento de las formas humificadas siguiendo el modelo de gestión del cultivo de palma, en general es posible incrementar el contenido de $\mathrm{C}$ total y el de todas las formas de $\mathrm{C}$; el modelo del suelo cultivado con banano y mango puede ser utilizado como referencia. Es conveniente incrementar el $\mathrm{C}$ recalcitrante en los suelos cultivados.

\section{AGRADECIMIENTOS}

Al Departamento de Edafología y Química Agrícola de la Universidad de Santiago de Compostela (España), por su apoyo logístico, técnico y humano. A la Universidad del Magdalena por su apoyo a través de los recursos de capacidad instalada y tiempo asignado para el desarrollo de la investigación.

\section{LITERATURA CITADA}

Albaladejo, J., R. Ortiz, N. Garcia-Franco, A. Ruiz-Navarro, M. Almagro, J. Garcia-Pintado, and M. Martínez-Mena. 2013. Land use and climate change impacts on soil organic carbon stocks in semi-arid Spain. J. Soils Sedim. 13: 265-277.

Almendros, C. 2004. Investigaciones básicas sobre el origen y la estructura molecular de las formas estables de materia orgánica relacionadas con el proceso de secuestro de carbono en los suelos. Edafologia 11: 229-249. 
Brady, N. and R. Weil. 2002. The nature and properties of soils. Prentice-Hall. Upper Saddle River, NJ, USA.

Calvo de Anta, R., F. Macías y A. Riveiro. 1992. Aptitud agronómica de los suelos de la provincia de La Coruña (Cultivos, Pinos, Robles, Eucaliptos y Castaños). (Mapa escala 1:200.000). Diputación Provincial de La Coruña. España.

Carvajal, A. F., A. Feijoo, H. Quintero y M. A. Rondón. 2009. Carbono orgánico del suelo en diferentes usos del terreno de paisajes andinos colombianos. Rev. Cienc. Suelo Nutr. 9: 222-235.

Ghani, A., M. Dexter, and K. W. Perrot. 2003. Hot-water extractable carbon in soils: A sensitive measurement for determining impacts of fertilisation, grazing and cultivation. Soil Biol. Biochem 35: 1231-1243.

Griffith, S. M. and M. Schnitzer. 1989. Oxidative degradation of soil humic substances. pp. 69-98. In: M. H. B. Hayes, P. Mac-Carthy, R. L. Malcolm, and R. S. Swift (eds.). Humic substances II- In search of structure. Wiley. New York, NY, USA.

Guo, L. B. and R. M. Gifford. 2002. Soil carbon stocks and land use change: A meta analysis. Global Change Biol. 8: 345-360.

Hassink, J. 1997. The capacity of soils to preserve organic C and $\mathrm{N}$ by their association with clay and silt particles. Plant Soil 191: 77-87.

Hoyos, N. and N. Comerford. 2005. Land use and landscape effects on aggregate stability and total carbon of Andisols from the Colombian Andes. Geoderma 129: 268-278.

Ibrahim, M., C. Cuartas, G. Ponce, V. Vega, F. Casasola y J. Rojas. 2007. Almacenamiento de carbono en el suelo y la biomasa arbórea en sistemas de usos de la tierra en paisajes ganaderos de Colombia, Costa Rica y Nicaragua. Agroforestería Am. 45: 27-36.

IPCC (Intergovernmental Panel on Climate Change). 2013. Climate change 2013: The physical science basis. Contribution of working group $\mathrm{I}$ to the $\mathrm{f}$ if th assessment report of the Intergovernmental Panel on Climate Change. Cambridge University Press. Cambridge, UK.

IGAC (Instituto Geográfico Agustín Codazzi). 2006. Métodos analíticos del laboratorio de suelos. IGAC. Bogotá, Colombia. ISBN: 9789589067987.

IGAC (Instituto Geográfico Agustín Codazzi). 2009. Estudio general de suelos y zonificación de tierras del Departamento de Magdalena. IGAC. Bogotá, Colombia. ISBN: 7703476003829.

Jiménez-Heredia, Y., C. M. Martínez-Bravo y N. J. ManceraRodríguez. 2010. Caracteristicas físicas y químicas del suelo en diferentes sistemas de uso y manejo en el Centro agropecuario Cotové, Santa Fé de Antioquia, Colombia. Rev. Suelos Ecuat. 40: 176-188.

Lal, R. 2010. Managing soils and ecosystems for mitigating anthropogenic carbon emissions and advancing global food security. Bioscience 60: 708-721. doi: 10.1525/bio.2010.60.9.8.

Lal, R. 2014. World soils and the carbon cycle in relation to climate change and food security. pp. 31-66. In: J. Weigelt, A. Müller, C. Beckh, K. Töpfer (eds.). Soils in the Nexus. Oekom Verlag, Müchen, Germany. ISBN 978-3-86581-431-9.

Lorenz, K. and R. Lal. 2014. Soil organic carbon sequestration in agroforestry systems. A review. Agron. Sustain. Dev. 34: 443-454. doi: 10.1007/s13593-014-0212-y.
Macías, F. 2004. Sumideros de carbono para el forzamiento climático antropoceno. Una visión de alternativas de actuación desde la ciencia del suelo. Edafologia 11: 7-25.

Macias, F., R. M. Calvo de Anta, L. Lado, R. Verde, X. Pena y M. Camps Arbestain. 2004. El sumidero de carbono de los suelos de Galicia. Edafologia 11: 341-376.

Malagon Castro, D. 2003. Ensayo sobre tipología de suelos colombianos; énfasis en génesis y aspectos ambientales. Rev. Acad. Colomb. Cienc. 27: 319-341. ISSN 0370-3908.

Montaño, N. M., F. Ayala, S. H. Bullock, O. Briones, F. García O., R. García S., Y. Maya, Y. Perroni, C. Siebe, Y. Tapia T., E. Troyo y E. Yépez. 2016. Almacenes y flujos de carbono en ecosistemas áridos y semiáridos de México: Síntesis y perspectivas. Terra Latinoamericana 34: 39-59.

Moreno, F. H. y W. Lara. 2003. Variación de carbono orgánico del suelo en bosques primarios intervenidos y secundarios. pp. 189-213. In: S. A. Orrego, J. I. Del Valle y F. H. Moreno (eds.). Medición de la captura de carbono en ecosistemas forestales tropicales de Colombia: contribuciones para la mitigación del cambio climático. Universidad Nacional de Colombia. Departamento de Ciencias Forestales. CAEMA. Bogotá D.C., Colombia.

Mosquera, O., P. Buurman, B. L. Ramirez, and M. C. Amezquita. 2012. Carbon stocks and dynamics under improved tropical pasture and silvopastoral systems in Colombian Amazonia. Geoderma 189-190: 81-86.

Parssons, J. W. 1989. Hydrolytic degradations of humic substances. pp. 99-109. In: M. H. B. Hayes, P. MacCarthy, R. L. Malcolm, and R. Swift (eds.). Humic substances II. In search of structure. Wiley. Chichester. UK.

Paul, E., S. J. Morris, R. T. Conant, and A. F. Plante. 2006. Does the acid hydrolysis-incubation method measure meaningful soil organic carbon pools? Soil Sci. Soc. Am. J. 70: 1023-1035.

Quéré, L. C., G. P. Peters, R. J. Andres, R. M. Andrew, T. A. Boden, P. Ciais, P. Friedlingstein, R. A. Houghton, G. Marland, R. Moriarty, S. Sitch, P. Tans, A. Arneth, A. Arvanitis, D. C. E. Bakker, L. Bopp, J. G. Canadell, L. P. Chini, S. C. Doney, A. Harper, I. Harris, J. I. House, A. K. Jain, S. D. Jones, E. Kato, R. F. Keeling, K. Klein Goldewijk, A. Körtzinger, C. Koven, N. Lefèvre, F. Maignan, A. Omar, T. Ono, G.-H. Park, B. Pfeil, B. Poulter, M. R. Raupach, P. Regnier, C. Rödenbeck, S. Saito, J. Schwinger, J. Segschneider, B. D. Stocker, T. Takahashi, B. Tilbrook, S. van Heuven, N. Viovy, R. Wanninkhof, A. Wiltshire, and S. Zaehle. 2014. Global carbon budget 2013. Earth Syst. Sci. Data 6: 235-263.

Ridley, A. M., K. R. Helyar, and W. J. Slattery. 1990. Soil acidification under subterranean clover (Trifolium subterraneaum L.) pastures in North-Eastern Victoria. Aust. J. Exp. Agric. 30: 195-201.

Shoji, S. and Y. Fujiwara. 1984. Active aluminium an iron in the humus horizons of andosols from northeastern Japan: Their forms, properties and significance in clay weathering. Soil Sci. 137: 216-226.

Schlesinger, W. H. and E. Bernhardt. 2013. Biogeochemistry: Analysis of global change. Elsevier. Oxford, UK. ISBN: 9780-12-385874-0.

Six, J., R. T. Conant, E. A. Paul, and K. Paustian. 2002. Stabilization mechanisms of soil organic matter: Implications for C-saturation of soils. Plant Soil 241: 155-176. 
Smith, P. 2008. Soil organic carbon dynamics and land-use change. pp. 9-22. In: A. K. Braimoh and P. L. G. Vlek (eds.). Land use and soil resources. Springer Netherlands. New York, NY, USA.

Stevenson, F. J. 1994. Humus chemistry. Genesis, composition, reactions. Wiley. New York, NY, USA. ISBN: 978-0-47159474-1.

Stockmann, U., M. A. Adams, J. W. Crawford, D. J. Field, N. Henakaarchchi, M. Jenkins, B. Minasny, A. B. McBratney, V. de R. de Courcelles, K. Singh, I. Wheeler, L. Abbott, D. A. Angers, J. Baldock, M. Bird, P. C. Brookes, C. Chenu, J. D. Jastrow, R. Lal, J. Lehmann, A. G. O’Donnell, W. J. Parton, D. Whitehead, and M. Zimmermann. 2013. The knowns, known unknowns and unknowns of sequestration of soil organic carbon. Agric. Ecosyst. Environ. 164: 80-99.

Tan, Z. and R. Lal. 2005. Carbon sequestration potential estimates with changes in land use and tillage practice in Ohio, USA. Agric. Ecosyst. Environ. 111: 140-152.

Tirol-Padre, A. and J. K. Ladha. 2004. Assessing the reliability of permanganate-oxidizable carbon as an index of soil labile carbon. Soil Sci. Soc. Am. J. 68: 969-978.
Vance, E. D., P. C. Brookes, and D. S. Jenkinson. 1987. An extraction method for measuring soil microbial biomass. Soil Biol. Biochem. 19: 703-707.

Vásquez Polo, J. R., D. Baena Garcia y J. C. Menjivar Flores. 2010. Variabilidad espacial de propiedades físicas y químicas en suelos de la granja experimental de la Universidad del Magdalena (Santa Marta, Colombia). Acta Agronóm. 59: 449-456.

Vásquez P., J. R., F. Macías V. y J. C. Menjivar F. 2011. Formas de carbono orgánico en suelos con diferentes usos en el departamento del Magdalena (Colombia). Acta Agronóm. 60: 369-379.

Velasco M., M., J. A. Almeida, P. Vidal T. y F. Macias. 2010. Fraccionamiento químico de carbono en Acrisolas con horizontes sómbricos del Sur de Brasil. Rev. Cienc. Agrarias 33: 277-286.

Von Lützow, M., I. Kögel-Knabner, K. Ekschmitt, H. Flessa, G. Guggenberger, E. Matzner, and B. Marschner. 2007. SOM fractionation methods: Relevance to functional pools and to stabilization mechanisms. Soil Biol. Biochem. 39: 2183-2207. 